\title{
CMEARTICLE Cytochrome P450 drug interactions with statin therapy
}

\author{
Ivanna Xin Wei Goh ${ }^{1}$, MBBS, Choon How $\underline{H o w}^{2}$, MMed, FCFP, Subramaniam Tavintharan $^{3,4}$, FRCP, FAMS
}

Mrs Tan was brought by her daughter to your clinic due to severe lethargy and body aches a week after her discharge from the hospital for chest infection. The 72-year-old Mrs Tan was not her usual energetic and chirpy self at this consultation, as compared to her regular visits for hypertension, hyperlipidaemia and gout. You reviewed the patient's discharge summary and medications, and telephoned her attending team to discuss her condition. Medication reconciliation revealed that she was currently on clarithromycin, colchicine and lovastatin, which all affected the cytochrome P450 3A4 (CYP3A4) pathway. You then presented to Mrs Tan and her daughter your joint recommendations for the patient to be readmitted for further testing and management.

\section{INTRODUCTION}

Statins are widely used in the treatment of hyperlipidaemia. Common side effects associated with statin therapy include headache, gastrointestinal symptoms and musculoskeletal complaints. These are usually mild, transient and nonspecific. However, statins have the potential to cause myopathy and rhabdomyolysis, which may be induced by a complex interaction among drugs, diseases and genetics. While the mechanism is not completely understood, the clinical association appears to be dose-dependent and higher with specific drug interactions. Although rhabdomyolysis is rare, it is important to note that a significant proportion of patients on statin therapy do experience musculoskeletal complaints. Although there are no fixed terminologies used to describe muscle toxicity, the American College of Cardiology/American Heart Association/National Heart, Lung and Blood Institute (ACC/AHA/NHLBI) Clinical Advisory ${ }^{(1)}$ has summarised the definitions of muscle symptoms, as shown in Table I.

\section{INTERACTIONS OF CYTOCHROME P450 INHIBITORS AND INDUCERS WITH STATINS}

About half of all drugs currently available in clinical practice are biotransformed in the liver, primarily by the cytochrome P450 (CYP450) system. The CYP450 system is also responsible for the microsomal metabolism of statins. The CYP3A4 isoenzyme is responsible for the metabolism of lovastatin, simvastatin and atorvastatin. Apart from an excess intake of grapefruit juice, concomitant use of drugs such as macolide antibiotics (e.g. erythromycin, clarithromycin), azole antifungals (e.g. fluconazole, ketoconazole and itraconazole) and immunosuppressive drugs
Table I. ACC/AHA/NHLBI definitions of muscle toxicity (2002). ${ }^{(2)}$

\begin{tabular}{ll}
\hline Muscle disorder & Symptom \\
\hline Myopathy & $\begin{array}{l}\text { Any disease of the muscles (acquired or } \\
\text { inherited) }\end{array}$ \\
Myalgia & $\begin{array}{l}\text { Muscle ache or weakness without elevation in } \\
\text { creatine kinase }\end{array}$ \\
Myositis & $\begin{array}{l}\text { Muscle symptoms with increased creatine } \\
\text { kinase levels }\end{array}$ \\
Rhabdomyolysis & $\begin{array}{l}\text { Muscle symptoms and creatine kinase > 10 } \\
\text { times the upper limit of normal, with elevation } \\
\text { in creatinine level (usually with brown urine and } \\
\text { urinary myoglobin) }\end{array}$ \\
\hline
\end{tabular}

(e.g. cyclosporine) causes competitive inhibition at the enzymatic level, resulting in higher serum levels of statins. Strong CYP3A4 inhibitors like protease inhibitors (e.g. ritonavir), itraconazole and ketoconazole can increase statin concentrations by up to 20-folds, thereby resulting in a greater risk of myositis and rhabdomyolysis. ${ }^{(1)}$ Macrolides and calcium channel blockers (e.g. amlodipine) may also increase the concentration of simvastatin by 4 - to 12 -fold. ${ }^{(3)}$

Fluvastatin and rosuvastatin, metabolised by a different CYP2C9 isoenzyme, are thought to be less subject to these interactions. Nevertheless, some patients taking fluvastatin have been reported to have an increased incidence of transaminitis and musculoskeletal effects when the drug was coadministered with cyclosporine. ${ }^{(1)}$ Caution should also be exercised when these statins are used with warfarin and protease inhibitors. ${ }^{(3,4)}$ Table II shows the inhibitors and inducers of the CYP450 enzymatic pathway.

\footnotetext{
${ }^{1}$ National Healthcare Group Polyclinics - Woodlands, ${ }^{2}$ SingHealth Polyclinics - Sengkang, ${ }^{3}$ Diabetes Centre, ${ }^{4}$ Division of Endocrinology, Khoo Teck Puat Hospital Singapore

Correspondence: Dr Ivanna Goh Xin Wei, Family Medicine Resident, National Healthcare Group Polyclinics, Woodlands Polyclinic, 10 Woodlands Street 31 Singapore 738579. ivanna.goh@mohh.com.sg
} 
Table II. Inhibitors and inducers of cytochrome P450 enzymatic pathway [adapted from Bellosta et al, 2004(3)].

\begin{tabular}{|c|c|c|}
\hline CYP substrates (statins) & Inhibitors/substrates & Inducers \\
\hline $\begin{array}{l}\text { CYP3A4 } \\
\text { (atorvastatin, lovastatin, } \\
\text { simvastatin) }\end{array}$ & $\begin{array}{l}\text { Ketoconazole, itraconazole, fluconazole, erythromycin, } \\
\text { clarithromycin, tricyclic antidepressants, nefazodone, } \\
\text { venlafaxine, fluvoxamine, fluoxetine, setraline, } \\
\text { cyclosporine A, tacrolimus, mibefradil, diltiazem, } \\
\text { verapamil, protease inhibitors, midazolam, } \\
\text { corticosteroids, grapefruit juice, tamoxifen, } \\
\text { amiodarone, warfarin }\end{array}$ & $\begin{array}{l}\text { Phenytoin, phenobarbital, barbiturates, rifampicin, } \\
\text { dexamethasone, cyclophosphamide, carbamazepine, } \\
\text { omeprazole, pioglitazone }\end{array}$ \\
\hline $\begin{array}{l}\text { CYP2C9 } \\
\text { (fluvastatin, rosuvastatin) }\end{array}$ & $\begin{array}{l}\text { Ketoconazole, fluconazole, sulfaphenazole, warfarin, } \\
\text { protease inhibitors }\end{array}$ & Rifampicin, phenobarbital, phenytoin \\
\hline
\end{tabular}

\section{Colchicine and statins}

Separately, colchicine and statins are both well known to cause myopathy. This interaction is more common in the elderly and those with renal insufficiency. Coadministration of both drugs may exacerbate the myotoxic effect. Colchicine is excreted by the hepatobiliary and renal systems. The liver is dependent on the availability of the CYP3A4 isoenzyme when it demethylates colchicine before excretion. Statins metabolised by the CYP3A4 pathway compete with colchicine for the CYP3A4 isoenzyme, which may result in higher serum concentrations of colchicine and statins, leading to a higher risk of myopathy. Several case studies have shown that the discontinuation of statins and colchicine led to a resolution of symptoms and re-administration of colchicine without statins did not result in any elevation in creatine kinase (CK), or myopathy. ${ }^{(2,5)}$ For practical purposes, other statins not metabolised by the CYP3A4 pathway (e.g. fluvastatin and rosuvastatin) should be considered when used with colchicine.

\section{Fibrates and statins}

Statins with fibrates deserve particular attention because myopathy can occur with either drug alone, and they may be additive when used together. A review of 36 published clinical trials involving the statin-fibrate combination therapy found that the prevalence of myopathy was $0.12 \% \cdot{ }^{(3)}$ In particular, the risk of developing of myopathy is mainly described with gemfibrozil and not fenofibrate. Gemfibrozil competitively inhibits CYP2C8 and also inhibits glucuronidation of statins, whereas fenofibrate is an inducer of both CYP3A4 and CYP2C8. This difference may explain the relative safety of fenofibrate versus gemfibrozil when used together with statins. The statin-gemfibrozil combination is contraindicated, while combination with fenofibrate may be considered for refractory or mixed hyperlipidaemia with severe hypertriglyceridaemia, especially in the presence of high cardiovascular risks.

\section{Genetic differences}

The SEARCH Collaborative Group carried out a genome-wide association study in 2008, and found that patients with a singlenucleotide polymorphism in SLCO1B1 located in chromosome 12 (which mediates the hepatic uptake of statins) had 4.5 times increased risk of myopathy in heterozygotes and 17.5 times increased risk in homozygotes when taking statins. ${ }^{(6)}$ The prevalence of SLCO1B1 polymorphisms in our local population is not known, and such gene polymorphisms could, in part, explain the interindividual variations in the risk of statin-induced myopathy.

\section{HOW DO STATINS DAMAGE MUSCLE?}

The mechanism of statin-induced myopathy is unknown, although there are a number of hypotheses. Some have postulated decreased sacrolemmal cholesterol, while others have suggested that mitochondrial dysfunction, increased apoptosis secondary to a decrease in isoprenoids and coenzyme Q10 play a role in statin-induced myopathy.

\section{SAFETY OF STATINS IN SINGAPORE}

There are six different types of statins licensed in Singapore, namely simvastatin, atorvastatin, lovastatin, pravastatin, fluvastatin and rosuvastatin. As mentioned earlier, the risk of statin-induced myopathy or rhabdomyolysis could be due to drug interactions in the CYP450 system. This risk is also dose-dependent.

The SEARCH trial, published in 2010, randomised 12,064 subjects with a history of myocardial infarction to either 80 mg or $20 \mathrm{mg}$ simvastatin for a total of 12 months. Their results showed that subjects allocated $80 \mathrm{mg}$ simvastatin produced only $6 \%$ more reduction in low-density lipoprotein (LDL) cholesterol compared to subjects given $20 \mathrm{mg}$ simvastatin. However, there were significantly more cases of myopathy in patients taking $80 \mathrm{mg}$ simvastatin daily compared to those who took only $20 \mathrm{mg}(0.9 \%$ vs. $0.02 \%)$. In addition, $22(0.4 \%)$ patients in the $80 \mathrm{mg}$ simvastatin group developed rhabdomyolysis, whereas there was no case of rhabdomyolysis in the $20 \mathrm{mg}$ simvastatin group. ${ }^{(7)}$ Therefore, the United States of America Food and Drug Administration had issued an advisory against the use of simvastatin at higher doses, especially in patients with concomitant use of the drugs listed in Table II. There are also recent reports regarding uncommon cognitive side effects, increased blood glucose and glycosylated haemoglobin with the use of statins.

\section{APPROACHES TO STATIN USE IN PATIENTS WITH RAISED CK OR MYALGIAS}

The safety and cardiovascular benefits of statins support their use as first-line treatment for hyperlipidaemia. However, there should be appropriate awareness of and attention to the potential for myopathy or rhabdomyolysis with statin therapy, as well as their interactions with other drugs. 
When a patient first presents with myalgia, muscle weakness or raised CK, it is important to rule out symptoms of hypothyroidism, alcoholism, excessive exercise, intramuscular injections, multisystem disease, and intake of other drugs that may interfere or compete with statins for metabolism before the initiation of statin therapy (Table II). Usually, after the aforementioned conditions are ruled out, the patient's cardiovascular risk profile, lipid goals, severity of muscle symptoms and degree of CK elevation should influence clinical decision-making. ${ }^{(8)}$ Some suggested approaches are as follows:

1. In asymptomatic patients with CK level not more than three times the upper limit of normal (ULN), a low dose of a statin may be considered in order to reach cholesterol level goals. Testing of CK level in these patients should be repeated within two weeks of initiation of statin treatment. If the CK level is not rising, it should be checked again in 6-12 weeks if the patient remains asymptomatic, or earlier, if symptomatic. If the CK level remains stable, the statin may be continued and CK level re-checked within 12 months if asymptomatic. CK level should, however, be re-tested within two weeks if any medications in Table II are added, or if any symptoms develop.

2. In patients whose CK levels are more than ten times the ULN and/or have myoglobinuria or raised creatinine levels, the prescribed statins should be stopped immediately and elevated CK should be urgently managed so as to reduce the risk of secondary renal injury.

3. In those with moderate to severe myalgia while on statin, the statin treatment should be stopped regardless of CK level.

4. In patients who have mild to moderately elevated CK levels not exceeding five times the ULN and in those whose statin has been discontinued or reduced due to inability to tolerate higher doses, the following options may be considered to help achieve target levels of LDL cholesterol and non-highdensity lipoprotein cholesterol levels: (a) addition of ezetimibe and/or bile acid sequestrants (BAS), e.g. cholestyramine, to a statin dose that is tolerated by the patient. The expected additional LDL-lowering for ezetimibe is $15 \%-20 \%$, and up to $30 \%$ for the maximum dose of cholestyramine. ${ }^{(8)}$ However, higher doses of cholestyramine are difficult to achieve due to the side effects produced. Of note, BAS should be avoided in hypertriglyceridaemia; (b) administration of a low dose of high potency statin, e.g. rosuvastatin at $5 \mathrm{mg}$ daily, every other day or weekly. Studies have shown promising results, with increased tolerability and acceptable reduction in LDL cholesterol, even with low dose and increased dosing intervals. ${ }^{(8)}$ This can be considered in monotherapy or in combination with other lipid-modifying agents, depending on the lipid goals.

\section{CONCLUSION}

It is important to understand the different pharmacological properties and profiles of the various statins and their drug interactions, so that we can better manage our patients and prevent statin-induced complications. This understanding may help prescribers balance patients' risks, as well as determine if prescribers should advise stopping statin, reducing the dose of statin for short durations, or changing to an alternative statin, when other drugs such as macrolides and azoles are indicated.

During Mrs Tan's second admission, lovastatin was stopped and she was optimally hydrated to treat her raised CK level (3,192 U/L). Her renal function had remained normal. Two weeks after discharge, you reviewed the patient at your clinic. A re-test of her CK level showed that it was normal at $177 \mathrm{U} / \mathrm{L}$. As Mrs Tan had been on lovastatin for the past ten years, she preferred not to make any changes to her medication. After the patient and her family had been briefed on the potential risks of drug interactions, a decision was made to restart the patient on her previous dose of lovastatin $(20 \mathrm{mg}$ at night). She was provided with a memo detailing her history of rhabdomyolysis and drug interaction in case she had to see a different doctor. Mrs Tan's CK levels remained normal after six months of reinitiation of statin.

\section{TAKE HOME MESSAGES}

1. Statins are commonly used in our community for the treatment of hyperlipidaemia.

2. Statins are generally well-tolerated, although they have the potential to cause myopathy and rhabdomyolysis.

3. There is an increased risk of adverse events in the setting of high-dose statins, certain comorbidities and genetic variants, as well as when coadministered with drugs or food that may interfere with the CYP450 pathway of drug metabolism.

4. Many clinically significant drug-drug interactions of statins can be avoided by correct selection and dosing of drugs.

5. The severity of muscle symptoms and CK levels should direct clinical decision-making in terms of selection or cessation of statins and adjustment of the dosage of lipid-lowering drugs.

ABSTRACT Statins are commonly used in the treatment of hyperlipidaemia. Although the benefits of statins are well-documented, they have the potential to cause myopathy and rhabdomyolysis due to the complex interactions of drugs, comorbidities and genetics. The cytochrome P450 family consists of major enzymes involved in drug metabolism and bioactivation. This article aims to highlight drug interactions involving statins, as well as provide updated recommendations and approaches regarding the safe and appropriate use of statins in the primary care setting.

Keywords: colchicine, fibrates, genetic variants, rhabdomyolysis, safety 


\section{REFERENCES}

1. Pasternak RC, Smith SC Jr, Bairey-Merz CN, et al. ACC/AHA/NHLBI clinical advisory on the use and safety of statins. J Am Coll Cardiol 2002; 40:567-72.

2. Hsu WC, Chen WH, Chang MT, Chiu HC. Colchicine-induced acute myopathy in a patient with concomitant use of simvastatin. Clin Neuropharmacol 2002; 25:266-8.

3. Bellosta S, Paoletti R, Corsini A. Safety of statins: focus on clinical pharmacokinetics and drug interactions. Circulation 2004; 109 (23 Suppl 1):III50-7. Review.

4. Neuvonen PJ, Niemi M, Backman JT. Drug interactions with lipid-lowering drugs: mechanisms and clinical relevance. Clin Pharmacol Ther 2006; 80:565-81.
5. Sahin G, Korkmaz C, Yalcin AU. Which statin should be used together with colchicine? Clinical experience in three patients with nephrotic syndrome due to AA type amyloidosis. Rheumatol Int 2008; 28:289-91.

6. SEARCH Collaborative Group, Link E, Parish S, et al. SLCO1B1 variants and statin-induced myopathy - a genomewide study. N Engl J Med 2008; 359:789-99.

7. Study of the Effectiveness of Additional Reductions in Cholesterol and Homocysteine (SEARCH) Collaborative Group, Armitage J, Bowman L, et al. Intensive lowering of LDL cholesterol with $80 \mathrm{mg}$ versus $20 \mathrm{mg}$ simvastatin daily in 12,064 survivors of myocardial infarction: a doubleblind randomised trial. Lancet 2010; 376:1658-69.

8. Eckel R. Approach to the patient who is intolerant of statin therapy. J Clin Endocrinol Metab 2010; 95:2015-22. 


\section{SINGAPORE MEDICAL COUNCIL CATEGORY 3B CME PROGRAMME} (Code SMJ 201303A)

1. The side effects associated with statin are often severe and devastating.

2. Myopathy and rhabdomyolysis are not side effects of statins use.

3. Patients on statin therapy rarely experience any musculoskeletal complaints.

4. Less than one in ten drugs currently available in clinical practice is biotransformed by the CYP450 system.

5. Lovastatin, simvastatin and atorvastatin are metabolised by the same cytochrome isoenzyme.

6. Grapefruit juice, erythromycin, itraconazole or cyclosporine, when taken together with lovastatin, simvastatin or atorvastatin, can potentially result in higher serum levels of the statin.

7. Amlodipine, when taken together with lovastatin, simvastatin or atorvastatin, can potentially result in higher serum levels of the statin.

8. Fluvastatin and rosuvastatin, metabolised by a different CYP2C9 isoenzyme, are subjected to less drugto-drug interactions.

9. Coadministration of colchicine and statins may exacerbate the myotoxic effect.

10. Fluvastatin and rosuvastatin should be considered when there is a long-term indication for colchicine.

11. Gemfibrozil competitively inhibits CYP2C8 and also inhibits glucuronidation of statins, resulting in a higher potential for side effects when used together with a statin.

12. Fenofibrate is an inducer of both CYP3A4 and CYP2C8, which may explain the relative safety of fenofibrate versus gemfibrozil when used together with statins.

13. A patient with a single-nucleotide polymorphism located within SLCO1B1 is associated with more than four times increased risk of myopathy when taking statins.

14. The mechanism of statin-induced myopathy is currently unknown.

15. Patients taking $80 \mathrm{mg}$ of simvastatin may only achieve $6 \%$ more reduction in LDL cholesterol compared to patients taking $20 \mathrm{mg}$ simvastatin.

16. There are significantly more cases of myopathy in patients taking $80 \mathrm{mg}$ simvastatin daily compared to those taking $20 \mathrm{mg}$ simvastatin daily.

17. Patients with myalgia, muscle weakness or raised creatine kinase (CK) before initiation of statin may have hypothyroidism, alcoholism, excessive exercise, intramuscular injections or multisystem disease.

18. Statin therapy is contraindicated and should not be considered in asymptomatic patients whose CK levels are two times the upper limit of normal (ULN).

19. In patients on statins, whose CK levels are more than ten times the ULN and/or who have myoglobinuria or raised creatinine levels, statins should be stopped and the patient referred urgently for management to reduce the risk of secondary renal injury.

20. Ezetimibe, bile acid sequestrants and low-dose rosuvastatin (daily or weekly) are alternative therapy options for patients who are unable to tolerate their standard statin therapies.

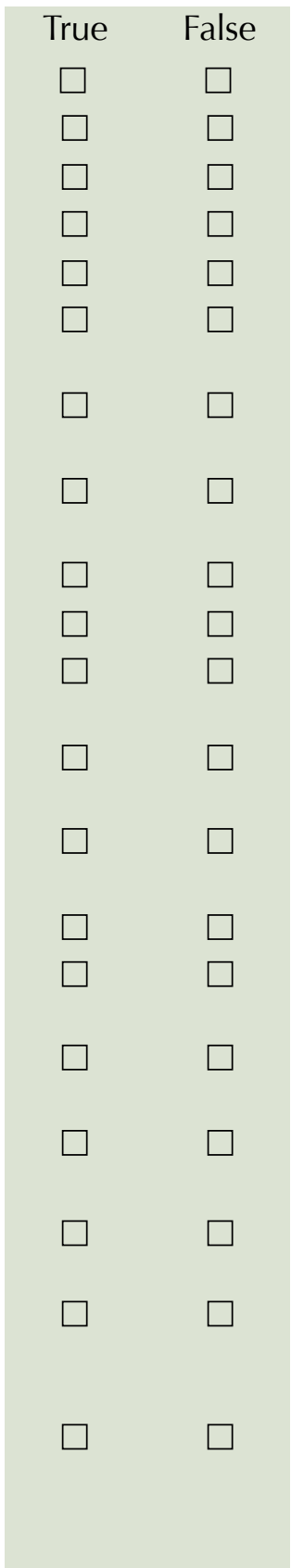

\section{Doctor's particulars:}

Name in full

MCR number

Specialty:

Email address

\section{SUBMISSION INSTRUCTIONS:}

(1) Log on at the SMJ website: http://www.sma.org.sg/publications/smjcurrentissue.aspx and select the appropriate set of questions. (2) Provide your name, email address and MCR number. (3) Select your answers and click "Submit".

\section{RESULTS:}

(1) Answers will be published in the SMJ May 2013 issue. (2) The MCR numbers of successful candidates will be posted online at the SMJ website by 29 April 2013. (3) Passing mark is $60 \%$. No mark will be deducted for incorrect answers. (4) The SMJ editorial office will submit the list of successful candidates to the Singapore Medical Council. (5) One CME point is awarded for successful candidates. 